
upf.
Universitat Department
Pompeu Fabra of Economics and Business
Barcelona

Economics Working Paper Series

Working Paper No. 1690

\title{
Optimal contracts with randomly arriving tasks
}

Daniel Bird and Alexander Frug

January 2020 


\title{
Optimal Contracts with Randomly Arriving Tasks*
}

\author{
Daniel Bird $\quad$ Alexander Frug
}

January 20, 2020

\begin{abstract}
Workers rarely perform exactly the same tasks every day. Instead, their daily workload may change randomly over time to comply with the fluctuating needs of the organization where they are employed. In this paper, we show that this typical randomness in workplaces has a striking effect on the structure of long-term employment contracts. In particular, simple intertemporal variability in the worker's tasks is sufficient to generate a rich promotion-based dynamics in which, occasionally, the worker receives a (permanent) wage raise and his future work requirements are reduced.
\end{abstract}

Keywords: Dynamic contracting, random tasks, seniority, promotion.

JEL Classification: D86, M51.

*Bird: Eitan Berglas School of Economics, Tel Aviv University (e-mail: dbird@tauex.tau.ac.il); Frug: Department of Economics and Business, Universitat Pompeu Fabra and Barcelona GSE (e-mail: alexander.frug@upf.edu). We are grateful to Larbi Alaoui, Jose Apesteguia, Eddie Dekel, Jan Eeckhout, Juan Jose Ganuza, Benny Moldovanu, Santiago Oliveros, Antonio Penta, Sandro Shelegia, and Joel Sobel for valuable suggestions and comments. The results in this paper were previously circulated as part of a manuscript titled "Monotone Contracts". 


\section{Introduction}

Promotions and seniority-based dynamics are widespread in workplaces. Modifications of work requirements and responsibilities, as well as wage raises, are often seen with tenure. In this paper, we link these observations to a feature that is common to many workplaces but has received scant attention in the literature: the tasks to which a worker is assigned change over time due to the varying short-term needs of the organization where he is employed. We show that simple variability in the worker's tasks generates a rich promotion-based dynamics. Most of the time, the worker's compensation does not depend on the realized workload. However, occasionally, the worker receives a permanent wage raise, and his future work requirements are reduced.

We analyze a simple and transparent model where different production opportunities arrive randomly in a manner that is i.i.d. across periods. Each period, the agent can exert effort whose productivity depends on the realized production opportunity. The principal incentivizes the agent via a periodic wage. We assume that the marginal productivity of effort, as well as the worker's marginal utility from the wage, are decreasing. Finally, we abstract away from frictions that arise from informational asymmetries and assume that both the worker's effort and the arrival of production opportunities are perfectly observed.

We show that, even though opportunities arrive according to a stationary distribution, the unique optimal employment contract consists of multiple hierarchical phases, which we interpret as different "ranks." Within each rank, the worker receives a fixed periodic wage. His workload, however, is stochastic since the required effort depends on the opportunities that are available in each period. Promotion to a new (higher) rank occurs upon the arrival of a production opportunity for which the worker's effort is more productive relative to all previous opportunities. On that occasion, the worker enjoys not only a permanent wage raise but also a reduction in his future job requirements. That is, from now on, whenever opportunities similar to those that have been available in the past arise again, the worker will be instructed to work less.

In general, as time goes by, the worker's expected within-period effort decreases while his periodic wage increases. Our analysis, therefore, offers new insights into wage ladders 
and seniority in workplaces by drawing a clear connection between the intertemporal variability of on-the-job assignments and the dynamics of effort and compensation. Specifically, sophisticated promotion dynamics may arise efficiently even in the absence of traditional frictions such as imperfect or asymmetric information about the worker's ability or actions, search frictions in the labor market, bargaining, accumulation of expertise, etc.

In addition to the aforementioned properties of optimal employment contracts, our results also provide a rationale for wage stickiness, and can thus be linked to the macroeconomics literature that studies the volatility of employment and productivity. Shimer (2005) showed that a reasonably calibrated textbook search and matching model (Diamond, 1982; Mortensen and Pissarides, 1994) cannot explain the volatility of the vacancy-unemployment ratio in the US data. In response, Hall (2005) argued that this puzzle can be resolved if wages are sticky. To support this line of argument, one might consider the varying opportunities in our model as "productivity shocks" of an aggregate nature. Since wages do not respond to negative productivity shocks under the optimal contract, we obtain a complete downward wage rigidity. Also, since wages respond to positive productivity shocks only when the productivity shock is unprecedentedly high, we obtain a partial upward wage rigidity. Thus, our model can provide a parsimonious theoretical foundation for the wage stickiness required to sustain Hall's argument that relies on nothing but the productivity shocks themselves and the firm's ability to offer long-term contracts. ${ }^{1}$

\subsection{Related Literature}

Many explanations have been proposed for the phenomenon of seniority in workplaces, and especially the increasing wage dynamics. For example, Becker (1962) and Parsons (1972) emphasize the effect of relationship-specific investment, Freeman and Medoff (1984) highlight the role of labor unions and collective bargaining, and Lazear (1981) and Carmichael (1983) illustrate how increasing wage dynamics emerge from informational frictions. More closely related to seniority-based dynamics are the papers that derive a downward wage rigidity in stochastic employment environments with symmetric information, Harris and

\footnotetext{
${ }^{1}$ Pissarides (2009) argues that wage stickiness can explain the "Shimer puzzle" only if the wage of a newly hired worker is sticky. Generically, aggregate productivity is not at its lowest when a worker is hired, and so our model predicts that the wage of a newly hired worker is sticky.
} 
Holmström (1982) study markets where there is uncertainty over the worker's skill, Holmström (1983) considers labor markets with stochastic demand, and Postel-Vinay and Robin (2002a,b) analyze labor markets with search frictions.

Our work differs from the strand of the literature discussed above in three key aspects. First, in addition to the dynamic structure of wage, our paper is informative about the dynamics of the worker's effort and the allocation of effort between various types of tasks along the worker's career. Second, in the previously mentioned papers, the downward wage rigidity is a result of competition for the worker's service. By contrast, the wage and effort dynamics in the present paper are entirely an outcome of within-interaction efficiency considerations (the worker's outside option is constant). Finally, in the previously mentioned papers, modifications in contract terms are triggered by purely exogenous events whereas, in our work, promotions are driven endogenously.

In a different strand of literature, Marcet and Marimon (1992) and Krueger and Uhlig (2006) study insurance markets with one-sided commitment. They show that the payment received by the insuree increases over time due to a mechanism similar to the one in Harris and Holmström (1982). In more recent work, Bird and Frug (2019a) and Forand and Zápal (2019) study dynamic project selection criteria in environments where projects arrive stochastically. $^{2}$ In those papers, projects are represented by pairs of payoffs, the players decide at what intensity to implement an available project, and the player's periodic payoffs are linear in the chosen intensity. Bird and Frug (2019a) consider a model where projects arrive according to a stationary distribution and their availability is privately observed by the agent. Their finding that is most related to this paper is that the initial selection criteria maximize the principal's utility and the final criteria maximize the agent's utility. However, the selection criteria do not evolve monotonically between these two extremes due to asymmetric information. By contrast, Forand and Zápal (2019) consider a model where projects arrive according to a more general process and their availability is publicly observed. Similar to the present paper, they find that the criteria evolve monotonically from the principal's to the agent's preferred criteria.

\footnotetext{
${ }^{2}$ Other papers that study project selection criteria include Armstrong and Vickers (2010), Li, Matouschek and Powell (2017), Samuelson and Stacchetti (2017), and Lipnowski and Ramos (2019).
} 
Finally, Ray (2002) and our companion paper, Bird and Frug (2019b), consider abstract contracting environments and derive general monotonicity results. Ray (2002) studies dynamic contracting in a repeated (deterministic) interaction with limited commitment and shows that the periodic contract converges over time to the agent's preferred contract. Bird and Frug (2019b), on the other hand, study contracting with full commitment in stochastic environments. They show that, in any contracting problem, certain components do not shift in the principal's favor over time under any optimal contract. Due to the generality of both papers, only a partial characterization of optimal contracts is derived that is not informative about important qualitative aspects. By contrast, the present paper imposes a structure that enables us to solve for the unique optimal contract and show that random arrival of production opportunities leads to a promotion-based dynamics.

The rest of the paper proceeds as follows. Section 2 presents the model, and Section 3 analyzes auxiliary settings that constitute the building block of the optimal contract. Section 4 contains the main result of the paper, and Section 5 concludes.

\section{Model}

We consider an infinitely repeated interaction between an agent and a principal. At the beginning of each period, nature chooses a task from the set $\mathcal{I}=\{1,2, \ldots, I\}$. Task $i \in \mathcal{I}$ is drawn with probability $q_{i}>0, \sum_{i=1}^{I} q_{i}=1$. The realized task (also referred to as the available task) is observed by both players. After observing which task has arrived, the agent chooses the effort $e \in[0, \infty)$ and, after observing this choice, the principal pays wage $w \in[0, \infty)$. The events in period $t$ are fully summarized by the triplet $\left(i_{t}, e_{t}, w_{t}\right)$.

We denote the principal's profit from effort on task $i \in \mathcal{I}$ by $\pi_{i}(\cdot)$, and the agent's utility from wage by $g(\cdot)$. We assume that the agent's and principal's periodic payoffs given the events $(i, e, w)$ are, respectively, $g(w)-e$ and $\pi_{i}(e)-w$. Moreover, we assume that the functions $g(\cdot), \pi_{1}(\cdot), \ldots, \pi_{I}(\cdot)$ are continuously differentiable, concave, strictly increasing, and satisfy $g(0)=\pi_{i}(0)=0$. In addition, we assume that tasks are ordered with respect to the marginal productivity of effort: for all $i<I$ and $e \in[0, \infty), \pi_{i}^{\prime}(e)<\pi_{i+1}^{\prime}(e)$. Finally, to avoid trivialities, we restrict attention to situations where all tasks are potentially profitable 
and it is suboptimal to incentivize infinite effort. To do so (due to the ordering assumption) it is sufficient to impose $\pi_{I}^{\prime}(0)>\frac{1}{g^{\prime}(0)}$ and $\lim _{e \rightarrow \infty} \pi_{1}^{\prime}(e)<\lim _{w \rightarrow \infty} \frac{1}{g^{\prime}(w)}$.

A sequence of triplets $h_{t}=\left\{\left(i_{s}, e_{s}, w_{s}\right)\right\}_{s<t}$, where, for all $s<t, i_{s} \in \mathcal{I}$ and $e_{s}, w_{s}$ are nonnegative numbers, represents a generic history at the beginning of period $t$. We denote by $H_{t}$ the set of all possible histories at the beginning of period $t$, by $H=\cup_{t \in \mathbb{N}} H_{t}$ the set of all "beginning-of-period" histories, by $\left(h_{t} ; i_{t}\right)$ a generic history at which the agent chooses the effort of period $t$, and by $\left(h_{t} ; i_{t}, e_{t}\right)$ a generic history at which the principal chooses the wage of period $t$. The players use the same factor $\delta<1$ to discount future periodic payoffs, and their objective is to maximize the discounted sum of periodic payoffs.

At the beginning of the interaction (prior to the realization of $i_{1}$ ) the principal proposes a contract, to which he is committed. A contract $\langle w o r k, p a y\rangle$ consists of two functions: a job description

$$
\text { work }: H \times \mathcal{I} \rightarrow[0, \infty)
$$

that specifies the required effort in period $t$ as a function of the history at the beginning of the period and the available task; and a compensation plan

$$
\text { pay }: H \times \mathcal{I} \times[0, \infty) \rightarrow[0, \infty)
$$

that specifies the agent's wage in period $t$ as a function of the history at the beginning of the period, the available task, and the agent's choice of effort.

The agent does not have commitment power. Thus, he will follow the contract only if doing so maximizes his expected discounted utility at every $\left(h_{t}, i_{t}\right)$. A contract $\langle w o r k$, pay $\rangle$ under which the agent finds it optimal to choose effort in accordance with $\operatorname{work}(\cdot)$ is called incentive compatible. For the rest of the paper we restrict attention to incentive-compatible contracts. Since the agent can always choose to exert no effort and his utility from wage is nonnegative, he can guarantee himself a continuation payoff of zero after any possible history. Therefore, an explicit individual rationality constraint is unneeded.

If we replace the concavity assumptions on $g(\cdot)$ and $\pi_{i}(\cdot)$ with their weak versions, the 
contract we construct in Section 4 remains an optimal contract, albeit not necessarily the unique optimal contract. For example, in the extreme case of linear utility from wage, a trivial optimal contract exists where the principal fully compensates the agent at the end of each period. While that contract seems natural in the case of linear utility, it cannot be approximated as a limit of optimal contracts where the agent's utility from wage is strictly concave. It is the strict concavity of the agent's utility that constitutes the link between different periods in our model. We now turn to constructing a contract that we later prove to be uniquely optimal.

\section{Auxiliary Problems}

The key step in the characterization of the optimal contract is to show that whenever the available task is better than all previously available tasks, there should be no debt carried over from the past (i.e., the agent's continuation utility should be zero). Given this insight (which we establish below), the whole interaction can be split into parts that can be analyzed separately. We now define $I$ auxiliary problems, one for each type of task, which constitute the building blocks of the optimal contract.

For $i \in \mathcal{I}$, let $P^{(i)}$ (referred to as "auxiliary problem $i$ ") denote the principal's optimization problem in an auxiliary setting where:

(1) At $t=1$ the realized task is of type $i$. In all other periods, task $j \in \mathcal{I}$ is realized with probability $q_{j}$.

(2) The interaction terminates upon the first arrival of a task that is better than $i$. Note that neither effort nor compensation can be provided after the arrival of such a task.

(3) The principal must hire the agent on fixed terms. A stationary contract $\left\langle\left(e_{j}\right)_{j \leq i}, w\right\rangle$ specifies the required effort $e_{j}$ whenever a task of type $j$ is available, for all $j \in\{1, . ., i\}$, and the fixed periodic wage of $w$ that the agent receives if he has followed the contract in the past. If the agent has not followed the contract in the past, his wage is zero.

For a contract to be incentive compatible in this auxiliary setting, the agent's cost of effort on the available task must not exceed his payoff from the current period's wage plus 
the expected discounted payoff from wage and effort in future periods. Let

$$
\lambda_{i}=1-\sum_{j>i} q_{j}
$$

denote the probability that the realized task is not better than $i$. Hence, the probability that the interaction in auxiliary problem $P^{(i)}$ is still ongoing in period $t+1$ is $\lambda_{i}^{t}$. The incentive-compatibility constraint when a task of type $j$ is available in auxiliary problem $P^{(i)}$ is

$$
e_{j} \leq g(w)+\sum_{t=2}^{\infty}\left(\lambda_{i} \delta\right)^{t-1}\left(g(w)-\frac{1}{\lambda_{i}} \sum_{k \leq i} q_{k} e_{k}\right)
$$

which simplifies to

$$
e_{j} \leq \frac{1}{1-\delta \lambda_{i}}\left(g(w)-\delta \sum_{k \leq i} q_{k} e_{k}\right) . \quad\left(I C_{j}^{(i)}\right)
$$

Thus, for $i \in \mathcal{I}$, auxiliary problem $P^{(i)}$ is given by

$$
P^{(i)}=\left\{\begin{array}{c}
\max _{w,\left(e_{j}\right)_{j \leq i}}\left[\pi_{i}\left(e_{i}\right)+\frac{1}{1-\delta \lambda_{i}}\left(\delta \sum_{j \leq i} q_{j} \pi_{j}\left(e_{j}\right)-w\right)\right] \\
\text { s.t. } \quad I C_{1}^{(i)}, \ldots, I C_{i}^{(i)}
\end{array}\right\} .
$$

Since $P^{(i)}$ is a convex optimization problem, it has a unique solution, which we denote by $\left\langle\left(e_{j}^{(i)}\right)_{j \leq i}, w^{(i)}\right\rangle$. We now derive several important properties of the solutions to the auxiliary problems; we will later use these properties to establish the optimality of the contract we construct.

Lemma 1. The only binding constraint in the solution to $P^{(i)}$ is $I C_{i}^{(i)}$.

Proof. In optimum, $w^{(i)}>0$ since $\pi_{i}^{\prime}(0)>\frac{1}{g^{\prime}(0)}$. Moreover, at least one of the incentivecompatibility constraints must be binding since, otherwise, slightly reducing $w^{(i)}$ would increase the value of the problem without violating any of the constraints.

Assume by way of contradiction that $I C_{j}^{(i)}$ is binding, for some $j<i$. This implies that $e_{j}^{(i)}>0$ and, since the right-hand side of all the constraints in $P^{(i)}$ are identical, it follows that $e_{j}^{(i)} \geq e_{i}^{(i)}$.

Consider the following modification for $\epsilon>0$ : decrease $e_{j}^{(i)}$ by $\epsilon \frac{1-\delta \lambda_{i}}{\delta q_{j}}$ and increase $e_{i}^{(i)}$ by $\epsilon\left(1+\frac{\delta q_{i}}{1-\delta \lambda_{i}}\right)^{-1}$. It is straightforward to verify that this modification does not increase 
the agent's expected discounted cost of effort when a type- $i$ task is available. In particular, it implies that the agent's expected discounted cost of effort decreases when any other type of task is available. Hence, this modification does not violate any of the constraints of $P^{(i)}$. The first-order effect of this modification on the value of the problem is

$$
\begin{aligned}
& \epsilon\left(\pi_{i}^{\prime}\left(e_{i}^{(i)}\right)\left(1+\frac{\delta q_{i}}{1-\delta \lambda_{i}}\right)^{-1}\left(1+\frac{\delta q_{i}}{1-\delta \lambda_{i}}\right)-\pi_{j}^{\prime}\left(e_{j}^{(i)}\right) \frac{1-\delta \lambda_{i}}{\delta q_{j}} \frac{\delta q_{j}}{1-\delta \lambda_{i}}\right) \\
& =\epsilon\left(\pi_{i}^{\prime}\left(e_{i}^{(i)}\right)-\pi_{j}^{\prime}\left(e_{j}^{(i)}\right)\right)>0
\end{aligned}
$$

where the inequality follows from the assumption that $e_{j}^{(i)} \geq e_{i}^{(i)}$ and the ordering of tasks. Thus, for a small enough $\epsilon$ this modification increases the value of the problem.

Lemma 1 implies that the solution to $P^{(i)}$ is given by the solution of the following Lagrangian function:

$$
\max _{\left(e_{j}\right)_{j \leq i}, w} \pi_{i}\left(e_{i}\right)+\frac{1}{1-\delta \lambda_{i}}\left(\delta \sum_{j \leq i} q_{j} \pi_{j}\left(e_{j}\right)-w\right)-\mu\left\{e_{i}-\frac{1}{1-\delta \lambda_{i}}\left(g(w)-\delta \sum_{j \leq i} q_{j} e_{j}\right)\right\}
$$

Lemma 2. For all $j \in\{1, \ldots, i\}, \pi_{j}^{\prime}\left(e_{j}^{(i)}\right) \leq \frac{1}{g^{\prime}\left(w^{(i)}\right)}$ with equality if $e_{j}^{(i)}>0$.

This lemma, which follows directly from the FOCs of the concave Lagrangian, stipulates that the marginal cost of periodic compensation is equal to the marginal productivity of effort from every implemented task. This intuitive result is less obvious than it seems. In particular, it relies on the fact that the auxiliary problem assumes the availability of its best admissible task in the initial period.

The next lemma ranks the agent's periodic compensation in the different auxiliary problems.

Lemma 3. The sequence $\left(w^{(1)}, w^{(2)}, \ldots, w^{(I)}\right)$ is strictly increasing.

This lemma is the linchpin of the promotion-based dynamics that arise in this model. To develop some intuition, suppose by way of contradiction that $w^{(i+1)} \leq w^{(i)}$ and note that the combination of Lemma 2 and the concavity of $\pi_{j}(\cdot)$ and $g(\cdot)$ would then imply that $e_{j}^{(i+1)} \geq e_{j}^{(i)}$, for all $j \leq i$. Now, consider the continuation of the interaction in auxiliary 
problem $P^{(i+1)}$, which begins with the arrival of a task of type $i$. From the arrival of that task to the first arrival of a better task (i.e., a task of type $l$, for some $l \geq i+1$ ), the agent exerts weakly more effort on all tasks and receives a weakly lower periodic compensation, compared to the solution to $P^{(i)}$. By Lemma 1 , none of the constraints $I C_{j}^{(i+1)}, j \leq i$, is binding in the solution to $P^{(i+1)}$. Therefore, a periodic compensation strictly lower than $w^{(i)}$ would suffice to incentivize (weakly) more effort than $\left\{e_{j}^{(i)}\right\}_{j=1}^{i}$ until the first arrival of a task that is better than task $i$. This contradicts the optimality of the solution to $P^{(i)}$.

Proof of Lemma 3. Suppose by way of contradiction that $w^{(i+1)} \leq w^{(i)}$. From the concavity of $g(\cdot)$ it follows that $g^{\prime}\left(w^{(i+1)}\right) \geq g^{\prime}\left(w^{(i)}\right)$. Thus, for all $j \leq i$, Lemma 2 and the concavity of $\pi_{j}(\cdot)$ imply that $e_{j}^{(i)} \leq e_{j}^{(i+1)}$.

The binding constraint of $P^{(i)}$ can be written as

$$
e_{i}^{(i)}\left(1-\delta \lambda_{i}\right)=g\left(w^{(i)}\right)-\delta \sum_{k \leq i} q_{k} \cdot e_{k}^{(i)} .
$$

Since $\lambda_{i+1}=\lambda_{i}+q_{i+1}$, we can similarly rewrite the binding constraint of $P^{(i+1)}$ as

$$
e_{i+1}^{(i+1)}\left(1-\delta \lambda_{i}\right)-\delta q_{i+1} \cdot e_{i+1}^{(i+1)}=g\left(w^{(i+1)}\right)-\delta \sum_{k \leq i} q_{k} \cdot e_{k}^{(i+1)}-\delta q_{i+1} \cdot e_{i+1}^{(i+1)}
$$

Adding $\delta q_{i+1} \cdot e_{i+1}^{(i+1)}$ to both sides, using the expression for the binding constraint of $P^{(i)}$ and the consequences of the assumption at the beginning of the proof, we get

$$
e_{i+1}^{(i+1)}\left(1-\delta \lambda_{i}\right)=g\left(w^{(i+1)}\right)-\delta \sum_{k \leq i} q_{k} \cdot e_{k}^{(i+1)} \leq g\left(w^{(i)}\right)-\delta \sum_{k \leq i} q_{k} \cdot e_{k}^{(i)}=e_{i}^{(i)}\left(1-\delta \lambda_{i}\right),
$$

which implies that $e_{i+1}^{(i+1)} \leq e_{i}^{(i)}$. Since $e_{i}^{(i)} \leq e_{i}^{(i+1)}$, it follows that $e_{i+1}^{(i+1)} \leq e_{i}^{(i+1)}$. However, as $\pi_{i+1}^{\prime}(e)>\pi_{i}^{\prime}(e)$ for all $e$, the only way this can occur without violating Lemma 2 is if $e_{i+1}^{(i+1)}=e_{i}^{(i+1)}=0$. This, in turn, implies that $e_{j}^{(i+1)}=0$ for all $j<i$, a solution that is not optimal due to our assumption that $\pi_{i}^{\prime}(0)>c^{\prime}(0)$.

The combination of Lemmas 2 and 3, together with the concavity assumptions, delivers some simple but important comparison results within a given auxiliary problem and across different problems. Higher effort is exerted on better tasks within each auxiliary problem, 
and lower effort is exerted on tasks of a particular type in the higher-indexed problem (one that starts with a better task) than on tasks of that same type in a lower-indexed problem. These results are the basis for the main qualitative properties of the unique optimal contract, which we will define shortly.

Corollary 1. Let $j \leq i$.

1. For $j>1, e_{j}^{(i)} \geq e_{j-1}^{(i)}$, with a strict inequality if $e_{j}^{(i)}>0$, and

2. For $i<I, e_{j}^{(i)} \geq e_{j}^{(i+1)}$, with a strict inequality if $e_{j}^{(i)}>0$.

\section{Main Result}

We now return to the original interaction and use the solutions of the auxiliary problems to define a particular contract, referred to as the phase mechanism. Later we prove that the phase mechanism is the essentially unique optimal contract. ${ }^{3}$

Definition of the phase mechanism.- For every $h_{t} \in H$ and $i_{t} \in \mathcal{I}$, let

$$
\mathscr{I}\left(h_{t} ; i_{t}\right)=\max \left\{i_{s}: s \leq t\right\}
$$

be the index of the best task that has been available at least once along $\left(h_{t} ; i_{t}\right)$. This function will intuitively correspond to the agent's rank under the phase mechanism. Note that this function does not decrease over time. The job description and the compensation-plan are, respectively,

$$
\operatorname{work}\left(h_{t}, i_{t}\right)=e_{i}^{\left(\mathscr{I}\left(h_{t} ; i_{t}\right)\right)}
$$

and

$$
\operatorname{pay}\left(h_{t}, i_{t}, e_{t}\right)= \begin{cases}w^{\left(\mathscr{G}\left(h_{t} ; i_{t}\right)\right)} & \text { if } e_{s}=\operatorname{work}\left(h_{s}, i_{s}\right) \text { for all } s \leq t \\ 0 & \text { otherwise }\end{cases}
$$

In words, the agent gets a positive wage only if he has followed his job description in all periods prior to the current payroll date. If he has done so, his wage and the required effort in every period are determined by the solution of the auxiliary problem indexed by the best

\footnotetext{
${ }^{3}$ We say that an optimal contract is essentially unique if all optimal contracts generate the same path of play for every realization of project arrivals. That is, the optimal contract is unique up to a redundant multiplicity that exists off the path of play.
} 
task that has been available so far.

Under the phase mechanism, the relationship between the principal and the agent can be described using the metaphor of a ratchet that allows advancement only in the direction that favors the agent (Lemma 3 and Corollary 1). That is, for any realization of task arrival, the periodic wage and the effort exerted on every type of task are given by monotonic step functions. When the periodic wage or effort requirements are updated, they jump to a new level where they stay until the next stochastic event causes another jump.

Proposition 1. The phase mechanism is the essentially unique optimal contract.

To develop some intuition for this result, consider the case of two possible types of tasks: a low-productivity task, $i=1$, and a high-productivity task, $i=2$. Suppose for a moment that the high-productivity task arrives in period 1 . In this case, the solution to $P^{(2)}$ specifies an optimal incentive-compatible contract that is stationary over time. ${ }^{4}$ Under this contract, the agent's expected payoff is zero and, as mentioned in Corollary 1, the effort exerted on a high-productivity task is higher than the effort exerted on a low-productivity task.

By construction, the periodic wage is identical in all periods. Therefore, while the agent's continuation utility is zero in periods in which a high-productivity task is available, his continuation utility is strictly positive whenever a low-productivity task is available. The positive continuation utility in these periods serves as an efficient compensation method for the high effort exerted on previous high-productivity tasks.

Now, suppose that the interaction does not begin with the high-productivity task. We refer to the time interval prior to the first arrival of the high-productivity task as phase 1 of the interaction. The stationary solution to $P^{(2)}$ is still an incentive-compatible contract (for the whole interaction); however, during phase 1 the agent strictly prefers not to deviate from the contract. Therefore, in this phase of the interaction, the principal can reduce the agent's periodic compensation and require more effort, without violating the agent's incentive-compatibility constraints. In optimum, the periodic wage is reduced and the effort

\footnotetext{
${ }^{4}$ In the proof we show that due to the concavity assumptions, on the path of play, the "within-phase" solutions are necessarily stationary and, thus, the solution to $P^{(2)}$ corresponds to the optimal contract if the high-productivity task arrives first.
} 
requirement is increased until the incentive-compatibility constraints in phase 1 are binding, while the marginal productivity of effort is kept equal to the marginal cost of compensation (the resulting wage and effort correspond to the solution to $P^{(1)}$ ).

As a result, in phase 1, the marginal productivity of effort and the marginal cost of compensation are lower than they are after the first arrival of the high-productivity task (phase 2). This leads to the key observation that any additional modification "between" phases is either unprofitable or infeasible. For example, it is not in the interest of the principal to incentivize additional effort in phase 1 by increasing compensation in phase 2 . On the other hand, even though the principal would benefit from increasing compensation in phase 1 in return for a higher effort on future high-productivity tasks in phase 2, doing so is not feasible as it would violate the agent's incentive-compatibility constraint at the beginning of phase 2 .

Proof of Proposition 1. First consider the auxiliary problems. For each $i \in \mathcal{I}$, define the relaxed version of $P^{(i)}$ to be identical to $P^{(i)}$ where requirement $(3)$ in the definition of the auxiliary setting of this problem is removed. We now show that the solution to $P^{(i)}$ and its relaxed version coincide. Consider the solution to $P^{(i)}$ and suppose that it is suboptimal under the relaxed version of $P^{(i)}$. Since the later is a convex maximization problem in which the objective function is separable in all arguments, there exists an improvement on the solution to $P^{(i)}$ of the following form: the required effort is modified at one particular history, and the compensation offered immediately afterwards (in the same period) is set at the lowest level under which all incentive-compatibility constraints are satisfied. By Lemma 2 , in the solution of $P^{(i)}$, the marginal benefit from every task equals the marginal cost of compensation (i.e., $\left.\frac{1}{g^{\prime}(\cdot)}\right)$. Since the agent's marginal utility from compensation and the marginal productivity of effort on any task are decreasing, every such modification strictly reduces the total expected value for the principal. Therefore, the solution to $P^{(i)}$ is the unique solution to the relaxed version of $P^{(i)}$.

Now consider the general environment. Denote by $C_{0}$ the class of all incentive-compatible contracts for which, whenever a task that is better than all previously available tasks arrives, the agent's continuation utility (before exerting effort) is zero. The phase mechanism is the unique optimal contract in the set $C_{0}$. To see this, notice that the restriction to contracts in $C_{0}$ implies that it is sufficient to show that the phase mechanism attains the highest 
expected value between any two (subsequent) earliest arrivals of tasks that are superior to all previously available ones. But this follows directly from the construction of the phase mechanism and the argument in the first step of the proof.

Finally, suppose that the phase mechanism is suboptimal in the class of all incentivecompatible contracts. Since the principal solves a convex optimization problem that is separable in all arguments, it follows from the claim established in the previous paragraph that there exists a profitable modification where (1) for some $\left(h_{t} ; i_{t}\right)$ in phase $k<I$, the phase mechanism is marginally altered in period $t$ in the direction that reduces the agent's periodic payoff (i.e., either the required effort is increased or the compensation is decreased), and $(2)$ at $\left(h_{t^{\prime}} ; i_{t^{\prime}}\right)$ which is part of phase $k^{\prime}>k$ and where $h_{t^{\prime}}$ is a continuation of $\left(h_{t} ; i_{t}\right)$, the phase mechanism is marginally changed to restore incentive compatibility. Since under the phase mechanism the marginal cost of compensation and the marginal benefit from effort during phase $k$ are below those of phase $k^{\prime}>k$, any such modification reduces the principal's expected payoff, a contradiction.

The optimal contract is essentially unique due to the concavity of the objective function and the convexity of the constraints.

\section{Conclusion}

We study a stylized dynamic contracting problem where the worker's tasks vary over time. Our main result shows that even when task arrival is i.i.d. across periods, the worker's career path exhibits seniority-based dynamics. As times goes by, the worker's expected wage increases while his expected workload decreases as a result of a rank-based contract.

Within each rank, the worker's wage is constant; however, his actual effort varies according to the random arrival of tasks. Upon the arrival of a task that is better than all previously available tasks, the worker is "promoted" to a new rank, he exerts less effort on tasks of lower quality (those that have been incentivized in the past), and his periodic wage is higher. Under this contract, promotions are not a consequence of new outside offers, additional information about the quality/productivity of the worker, a contest of any form, or an efficient back-loading of compensation to overcome informational frictions. Instead, promotions arise entirely as an efficient response to the common feature of variation in a 
worker's day-to-day tasks.

The rank structure of the optimal contract also implies that random shocks to the worker's productivity have a very limited impact on his wage and job requirements when the employer can offer long-term contracts. The simplest manifestation of this stickiness is that the arrival of any task that is worse than the task that was available in the period in which the worker was hired has no impact on his wage or job requirements. Thus, even the wage of a recently hired worker can exhibit considerable stickiness if he was hired in a period in which productivity was high. More generally, the worker's rank depends only on the best-to-date task and so, as time goes by, the probability that the realized task will change his rank decreases. Hence, as the worker's tenure at the firm increases so does the stickiness of his wage and work requirements.

\section{References}

Armstrong, Mark, and John Vickers. 2010. "A model of delegated project choice." Econometrica, 78(1): 213-244.

Becker, Gary S. 1962. "Investment in human capital: A theoretical analysis." Journal of Political Economy, 70(5): 9-49.

Bird, Daniel, and Alexander Frug. 2019a. "Dynamic nonmonetary incentives." American Economic Journal: Microeconomics, 11(4): 111-150.

Bird, Daniel, and Alexander Frug. 2019b. "Monotone contracts." Tel Aviv University. Mimeo.

Carmichael, Lorne. 1983. "Firm-specific human capital and promotion ladders." The Bell Journal of Economics, 14(1): 251-258.

Diamond, Peter A. 1982. "Aggregate demand management in search equilibrium." Journal of Political Economy, 90(5): 881-894.

Forand, Jean Guillaume, and Jan Zápal. 2019. "Production priorities in dynamic relationships." Mimeo. 
Freeman, Richard, and James Medoff. 1984. What Do Unions Do. New York:Basic Books.

Hall, Robert E. 2005. "Employment fluctuations with equilibrium wage stickiness." American Economic Review, 95(1): 50-65.

Harris, Milton, and Bengt Holmström. 1982. "A theory of wage dynamics." Review of Economic Studies, 49(3): 315-333.

Holmström, Bengt. 1983. "Equilibrium long-term labor contracts." Quarterly Journal of Economics, 98: 23-54.

Krueger, Dirk, and Harald Uhlig. 2006. "Competitive risk sharing contracts with onesided commitment." Journal of Monetary Economics, 53(7): 1661-1691.

Lazear, Edward P. 1981. "Agency, earnings profiles, productivity, and hours restrictions." American Economic Review, 71(4): 606-620.

Li, Jin, Niko Matouschek, and Michael Powell. 2017. "Power dynamics in organizations." American Economic Journal: Microeconomics, 9(1): 217-241.

Lipnowski, Elliot, and Joao Ramos. 2019. "Repeated delegation." Columbia University Mimeo.

Marcet, Albert, and Ramon Marimon. 1992. "Communication, commitment, and growth." Journal of Economic Theory, 58(2): 219-249.

Mortensen, Dale T., and Christopher A. Pissarides. 1994. "Job creation and job festruction in the theory of unemployment." The Review of Economic Studies, 61(3): 397415.

Parsons, Donald O. 1972. "Specific human capital: An application to quit rates and layoff rates." Journal of Political Economy, 80(6): 1120-1143.

Pissarides, Chrisopher A. 2009. "The unemployment volatility puzzle: Is wage stickiness the answer?" Econometrica, 77(5): 1339-1369.

Postel-Vinay, Fabien, and Jean-Marc Robin. 2002a. "The distribution of earnings in an equilibrium search model with state-dependent offers and counteroffers." International Economic Review, 43(4): 989-1016. 
Postel-Vinay, Fabien, and Jean-Marc Robin. 2002b. "Equilibrium wage dispersion with worker and employer heterogeneity." Econometrica, 70(6): 2295-2350.

Ray, Debraj. 2002. "The time structure of self-enforcing agreements." Econometrica, $70(2): 547-582$.

Samuelson, Larry, and Ennio Stacchetti. 2017. "Even up: Maintaining relationships." Journal of Economic Theory, 169: 170-217.

Shimer, Robert. 2005. "The cyclical behavior of equilibrium unemployment and vacancies." American Economic Review, 95(1): 25-49. 